\title{
A case study-based analysis of the influence of the fuzzy data shape in quantifying their Fréchet's variance
}

\author{
Sara de la Rosa de Sáa ${ }^{1}$, Carlos Carleos ${ }^{2}$, María Teresa López ${ }^{2}$, \\ and Manuel Montenegro ${ }^{2}$
}

\begin{abstract}
In previous studies it has been shown that assumming a trapezoidal shape to model fuzzy number-valued data is not statistically restrictive in case we focus on the (Aumann-type) means of these data. The assertion has been supported by both case and simulation studies. This paper aims to analyze by means of a case study whether the same assertion applies in dealing with the Fréchet-type variance. More concretely, the $p$-values of tests have been compared for trapezoidal assessment $v s$ other frequently used ones, like some $L R$ assessments. The analysis is illustrated and corroborated with a real-life example. This analysis indicates that the shape of the fuzzy assessment scarcely affects statistical conclusions.
\end{abstract}

\section{Introduction}

Trapezoidal fuzzy numbers have been shown to be an easy-to-use/draw choice to model data from intrinsically imprecise-valued magnitudes. This is issued to the ease to handle for most of the computations, the ease to understand their meaning, and the ease of their elicitation, especially when people assessing fuzzy numbers have a low expertise/background about.

Thus, trapezoidal fuzzy numbers are characterized by simply giving their core (interval of the real values which are considered to be 'fully compatible' with the valuation to be elicited) and their support (interval of the real values which are considered to be 'compatible to some extent' with the valuation to be elicited). The remaining values can be directly obtained by a kind of 'linear interpolation' of these two intervals.

In Lubiano et al. [8] it has been empirically shown that, when fuzzy datasets are summarized by their fuzzy-valued means, the considered data shape is mostly not statistical relevant.

${ }^{1}$ Oficina de Evaluación de Tecnologías Sanitarias, Servicio de Salud del Principado de Asturias, Spain, sara. delarosa@ sespa.es $\cdot{ }^{2}$ Departamento de Estadística, I.O. y D.M., Universidad de Oviedo, Spain, \{carleos, mt lopez, mmontenegro\}@uniovi.es 
In this paper we are going to check that the same conclusion can be drawn when fuzzy datasets are summarized by their real-valued variances. For this purpose, the real-life example analyzed in detail in Gil et al. [11] and Lubiano et al. [26, 8], and later recalled in Section 3, is considered for the comparative discussion. Some two-and $k$-samples hypothesis test about means $p$-values in Ramos-Guajardo and Lubiano [10] have been computed by considering all data as either being trapezoidal (as assumed in $[11,26,8]$ ) or belonging to any of the $L R$ classes recalled in the next section.

\section{Preliminaries}

A (bounded) fuzzy number (also referred to by some authors as a fuzzy interval) is an imprecise-valued amount that is formalized as a mapping $\widetilde{U}: \mathbb{R} \rightarrow[0,1]$ such that for all $\alpha \in[0,1]$, the $\alpha$-level set, defined as

$$
\widetilde{U}_{\alpha}= \begin{cases}\{x \in \mathbb{R}: \widetilde{U}(x) \geq \alpha\} & \text { if } \alpha \in(0,1] \\ \operatorname{cl}\{x \in \mathbb{R}: \widetilde{U}(x)>0\} & \text { if } \alpha=0\end{cases}
$$

with 'cl' denoting the closure of the set, is a nonempty compact interval. $\widetilde{U}(x)$ is intuitively interpreted as the 'degree of compatibility' of the real number $x$ with $\widetilde{U}$.

The space of (bounded) fuzzy numbers will be denoted by $\mathscr{F}_{c}^{*}(\mathbb{R})$.

A well-known and frequently used family of fuzzy numbers is that of trapezoidal fuzzy numbers. If $a, b, c, d \in \mathbb{R}$ with $a \leq b \leq c \leq d$, the trapezoidal fuzzy number $\operatorname{Tra}(a, b, c, d)$ is such that for each $\alpha \in[0,1]$ the $\alpha$-level set equals

$$
(\operatorname{Tra}(a, b, c, d))_{\alpha}=[a+\alpha(b-a), d+\alpha(c-d)] .
$$

A wider interesting family of fuzzy numbers, including the one of trapezoidal fuzzy numbers, is that of the $L R$-fuzzy numbers (see Dubois and Prade [5]) with $L$ and $R$ invertible functions. If $a, b, c, d \in \mathbb{R}$ with $a \leq b \leq c \leq d$, the $L R$-fuzzy number $\operatorname{LR}(a, b, c, d)$ is such that for each $\alpha \in[0,1]$ the $\alpha$-level set is such that

$$
\begin{aligned}
& \inf (L R(a, b, c, d))_{\alpha}=a+(b-a) L^{-1}(\alpha), \\
& \sup (L R(a, b, c, d))_{\alpha}=d-(d-c) R^{-1}(\alpha) .
\end{aligned}
$$

Along this work we are going to consider quadratic functions (the so-called $\Pi$-curves) and functions with parametric monotonic Hermite-type interpolation, either using $(2,2)$-rational splines $\left(L U_{1 A}\right.$ and $\left.L U_{1 B}\right)$ or mixed exponential splines ( $L U_{2 A}$ and $L U_{2 B}$ ) (see Figure 1) (see, for more details about Stefanini et al. [11]). 

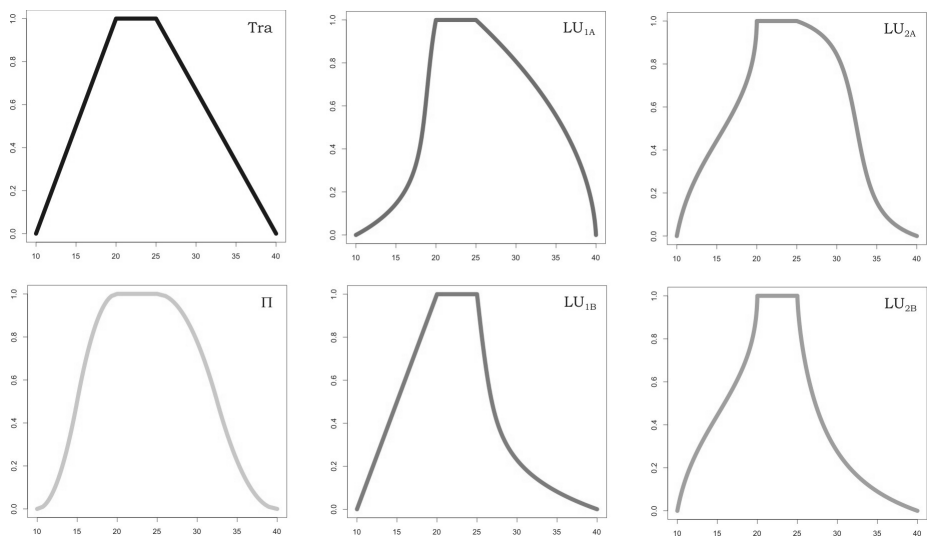

Fig. 1: Six types of fuzzy numbers sharing core [20,25] and support $(10,40)$ and differing in shape. On the left, trapezoidal (top) and $\Pi$-curve (bottom), along with four different $L R$-fuzzy numbers on the middle and the right

More specifically, if $\widetilde{U} \equiv L R(a, b, c, d)$, and $L R \in\left\{\operatorname{Tra}, \Pi, L U_{1 A}, L U_{1 B}, L U_{2 A}, L U_{2 B}\right\}$, then for each $\alpha \in[0,1]$

$$
\widetilde{U}_{\alpha}=\left[a+l_{L R}(\alpha)(b-a), c+r_{L R}(\alpha)(d-c)\right],
$$

where the functions involved in the left and right arms can be seen in detail in Table 1.

Table 1: Expressions for functions $l_{L R}$ and $r_{L R}$ in the horizontal view of $L R$-fuzzy numbers with $L R$ ranging on $\left\{\mathrm{Tra}, \Pi, L U_{1 A}, L U_{1 B}, L U_{2 A}, L U_{2 B}\right\}$

\begin{tabular}{|c|c|c|}
\hline$L U$ & $l_{L U}(\alpha)$ & $r_{L U}(\alpha)$ \\
\hline Tra & $\alpha$ & $1-\alpha$ \\
\hline \multirow{2}{*}{$\Pi$} & $\{\sqrt{\alpha / 2} \quad$ if $\alpha<1 / 2$ & $\{1-\sqrt{\alpha / 2}$ if $\alpha<1 / 2$ \\
\hline & $1-\sqrt{(1-\alpha) / 2}$ otherwise & $\sqrt{(1-\alpha) / 2}$ otherwise \\
\hline$L U_{1 A}$ & $\frac{\alpha^{2}+5 \alpha(1-\alpha)}{1+3.5 \alpha(1-\alpha)}$ & $(1-\alpha)(1+0.9 \alpha)$ \\
\hline$L U_{1 B}$ & $\alpha$ & $1-\frac{\alpha^{2}+5 \alpha(1-\alpha)}{1+3.2 \alpha(1-\alpha)}$ \\
\hline$L U_{2 A}$ & $\frac{\alpha^{2}(3-2 \alpha)-0.5(1-\alpha)^{1.55}+0.5+0.05 \alpha^{1.55}}{1.55}$ & $1-\frac{\alpha^{2}(3-2 \alpha)-5(1-\alpha)^{11}+5+5 \alpha^{11}}{11}$ \\
\hline$L U_{2 B}$ & $\frac{\alpha^{2}(3-2 \alpha)-0.5(1-\alpha)^{1.55}+0.5+0.05 \alpha^{1.55}}{1.55}$ & $1-\frac{\alpha^{2}(3-2 \alpha)-5(1-\alpha)^{6.05}+5+0.05 \alpha^{6.05}}{6.05}$ \\
\hline
\end{tabular}

Key tools for the statistical analysis of fuzzy data are the following:

- the arithmetic with fuzzy numbers;

- the metric between fuzzy numbers;

- the model for the random mechanism generating fuzzy data. 
Regarding the arithmetic, we will make use of the one based on Zadeh's extension principle [13].

Given $\widetilde{U}, \widetilde{V} \in \mathscr{F}_{c}^{*}(\mathbb{R})$, the sum of $\widetilde{U}$ and $\widetilde{V}$ is the fuzzy number $\widetilde{U}+\widetilde{V} \in \mathscr{F}_{c}^{*}(\mathbb{R})$ such that for each $\alpha \in[0,1]$

$$
(\widetilde{U}+\widetilde{V})_{\alpha}=\left[\inf \widetilde{U}_{\alpha}+\inf \widetilde{V}_{\alpha}, \sup \widetilde{U}_{\alpha}+\sup \widetilde{V}_{\alpha}\right] .
$$

Given $\widetilde{U} \in \mathscr{F}_{c^{*}}^{*}(\mathbb{R})$ and a scalar $\gamma \in \mathbb{R}$, the product of $\widetilde{U}$ by the scalar $\gamma$ is the fuzzy number $\gamma \cdot \widetilde{U} \in \mathscr{F}_{c}^{*}(\mathbb{R})$ such that for each $\alpha \in[0,1]$

$$
(\gamma \cdot \widetilde{U})_{\alpha}= \begin{cases}{\left[\gamma \cdot \inf \widetilde{U}_{\alpha}, \gamma \cdot \sup \widetilde{U}_{\alpha}\right]} & \text { if } \gamma \geq 0 \\ {\left[\gamma \cdot \sup \widetilde{U}_{\alpha}, \gamma \cdot \inf \widetilde{U}_{\alpha}\right]} & \text { otherwise }\end{cases}
$$

It can be easily proved that for fixed invertible functions $L$ and $R$, the family of $L R$-fuzzy numbers is closed under the sum and the product by scalars. More concretely,

$$
\begin{gathered}
L R(a, b, c, d)+L R\left(a^{\prime}, b^{\prime}, c^{\prime}, d^{\prime}\right)=L R\left(a+a^{\prime}, b+b^{\prime}, c+c^{\prime}, d+d^{\prime}\right), \\
\gamma \cdot \operatorname{LR}(a, b, c, d)= \begin{cases}L R(\gamma a, \gamma b, \gamma c, \gamma d) & \text { if } \gamma \geq 0 \\
L R(\gamma d, \gamma c, \gamma b, \gamma a) & \text { otherwise. }\end{cases}
\end{gathered}
$$

These two operations do not endow $\mathscr{F}_{c}^{*}(\mathbb{R})$ with a linear, but with a conical structure, so special care should be taken in attempting to extend and deal with difference between fuzzy numbers. Actually, some of the inconveniencies associated with the nonlinearity have been substantially overcome in developing statistics with fuzzy data by incorporating suitable distances between them. In this respect, the metric given below has been introduced by Bertoluzza et al. [1], and it is a quite convenient choice for the statistical developments.

Given $\widetilde{U}, \widetilde{V} \in \mathscr{F}_{c}^{*}(\mathbb{R})$ and $\theta \in(0,1]$, Bertoluzza et al.'s $\theta$-distance between $\widetilde{U}$ and $\widetilde{V}$ is the real number

$$
D_{\theta}(\widetilde{U}, \widetilde{V})=\sqrt{\int_{[0,1]}\left(\left[\operatorname{mid} \widetilde{U}_{\alpha}-\operatorname{mid} \widetilde{V}_{\alpha}\right]^{2}+\theta\left[\operatorname{spr} \widetilde{U}_{\alpha}-\operatorname{spr} \widetilde{V}_{\alpha}\right]^{2}\right) d \alpha} .
$$

The most common particular choices of the parameter $\theta$ are $\theta=1$ and $1 / 3$, since the first one corresponds to only taking into account and uniformly the squared distances between the extremes of the level sets, and the second one corresponds to taking into account and uniformly the squared distances between all the convex linear combinations of the extremes of the level sets.

Fuzzy number-valued data set in the case study to be considered, come from a so-called fuzzy rating scale (FRS), as introduced by Hesketh et al. [17], that allows a rater to draw the fuzzy number that best represents his/her score. The guideline for the mechanism to draw such a fuzzy number is as follows: 
Step 1. A reference bounded interval/segment is first considered. This is often chosen to be $[0,10]$ or $[0,100]$, but the choice of the interval is not at all a constraint. The end-points are often labeled in accordance with their meaning referring to the degree of agreement, satisfaction, quality, and so on.

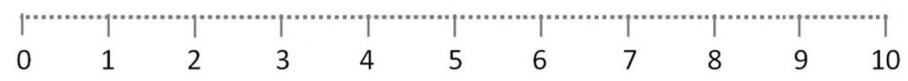

Step 2. The core, or 1-level set, associated with the response is determined. It corresponds to the interval consisting of the real values within the reference one which are considered to be as 'fully compatible' with the response.

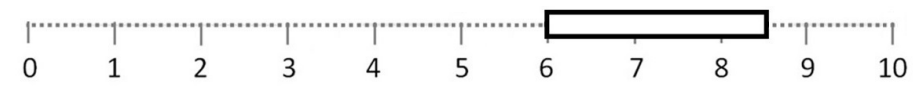

Step 3. The support, or its closure or 0-level set, associated with the response is determined. It corresponds to the interval consisting of the real values within the referential that are considered to be as 'compatible to some extent' with the response, and it should be always included in the reference interval.

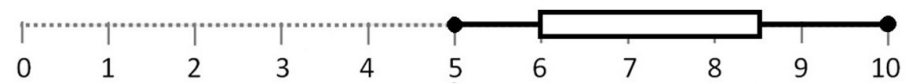

Step 4. The two intervals are 'interpolated' to get a fuzzy number. For instance, if a linear interpolation is considered a trapezoidal fuzzy number is obtained.

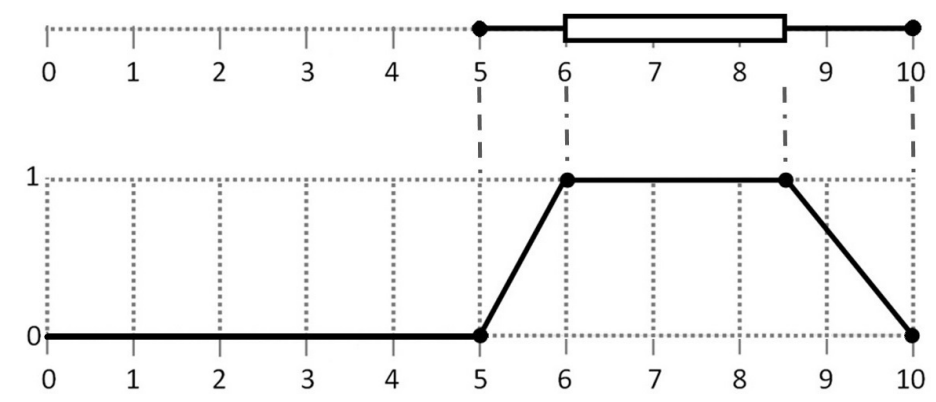

In developing statistics with fuzzy data coming from intrinsically imprecisevalued attributes, random fuzzy numbers constitute a well-formalized model within the probabilistic setting for the random mechanisms generating such data. Random fuzzy numbers, as defined by Puri and Ralescu [9] (in a more general context), integrate randomness (associated with the data generation) and fuzziness (associated with data nature). 
Given a probability space $(\Omega, \mathscr{A}, P)$, an associated random fuzzy number (for short RFN) is a mapping $\mathscr{X}: \Omega \rightarrow \mathscr{F}_{c}^{*}(\mathbb{R})$ such that for all $\alpha \in[0,1]$ the intervalvalued mapping $\mathscr{X}_{\alpha}$ is a compact random interval (i.e., the real-valued mappings $\inf \mathscr{X}_{\alpha}$ and $\sup \mathscr{X}_{\alpha}$ are real-valued random variables). Equivalently, a mapping $\mathscr{X}: \Omega \rightarrow \mathscr{F}_{c}^{*}(\mathbb{R})$ is said to be an RFN if and only if it is a Borel-measurable mapping w.r.t. the Borel $\sigma$-field generated on $\mathscr{F}_{c}^{*}(\mathbb{R})$ by the topology induced by $D_{\theta}^{\varphi}$; this Borel-measurability ensures that one can properly refer to the distribution induced by an RFN, the stochastic independence of RFN's, and so on, without needing to state expressly these notions.

In summarizing the induced distribution of a random fuzzy number, two measures/parameters are the most commonly used, namely, the Aumann-type mean (see Puri and Ralescu [9]) and the Fréchet-type variance (see, for instance, Lubiano et al. [6]).

Given an RFN $\mathscr{X}$ associated with the probability space $(\Omega, \mathscr{A}, P)$, the (population) Aumann-type mean of $\mathscr{X}$ is the fuzzy number $\widetilde{E}(\mathscr{X}) \in \mathscr{F}_{c}^{*}(\mathbb{R})$, if it exists, such that for each $\alpha \in[0,1]$

$$
(\widetilde{E}(\mathscr{X}))_{\alpha}=\left[E\left(\inf \mathscr{X}_{\alpha}\right), E\left(\sup \mathscr{X}_{\alpha}\right)\right]
$$

with $E$ denoting the expected value of a real-valued random variable.

If $\mathscr{X}$ is an $L R$-valued random fuzzy number for fixed invertible functions $L$ and $R$, then $\widetilde{E}(\mathscr{X})=L R\left(E\left(\inf \mathscr{X}_{0}\right), E\left(\inf \mathscr{X}_{1}\right), E\left(\sup \mathscr{X}_{1}\right), E\left(\sup \mathscr{X}_{0}\right)\right)$.

The Aumann-type mean preserves the main valuable properties from the realvalued case (i.e., additivity, equivariance under affine transformations, coherence with the above-described fuzzy arithmetic, and support by SLLN's).

In extending the variance of real-valued random variables to RFN's, Fréchet's approach has been considered, so that it can be interpreted as a measure of the 'least squares error/distance' in approximating the values of the RFN by a (non-random) fuzzy number. by

The (population) Fréchet-type variance is the real number $\sigma_{\mathscr{X}}^{2}$, if it exists, given

$$
\sigma_{\mathscr{X}}^{2}=E\left(\left[D_{\theta}(\mathscr{X}, \widetilde{E}(\mathscr{X}))\right]^{2}\right)=\int_{[0,1]} \operatorname{Var}\left(\operatorname{mid} \mathscr{X}_{\alpha}\right) d \alpha+\theta \cdot \operatorname{Var}\left(\operatorname{spr} \mathscr{X}_{\alpha}\right) d \alpha
$$

The Fréchet variance of an RFN satisfies the usual properties for this concept (i.e., nonnegativity, and vanishment for degenerate RFN's, invariance under translation, and additivity under independence of the involved RFN's).

\section{Case study to be analyzed}

The case study to be analyzed is related to the well-known questionnaire TIMSSPIRLS 2011 which is conducted on the population of Grade 4 students (i.e., nine to ten years old) and concerns their opinion and feeling on aspects regarding reading, 
math, and science. This questionnaire is rather standard and most of the involved questions have to be answered according to a 4-point Likert scale, responses being DisAGREE A LOT, DISAGREE A LITTLE, AGREE A LITTLE, and AGREE A LOT.

To get more expressive responses and informative conclusions, the original questionnaire form has been adapted to allow a double-type response: the original Likert and a fuzzy rating scale-based one with reference interval $[0,10]$ (see Figure 2 for one of the items in the questionnaire).

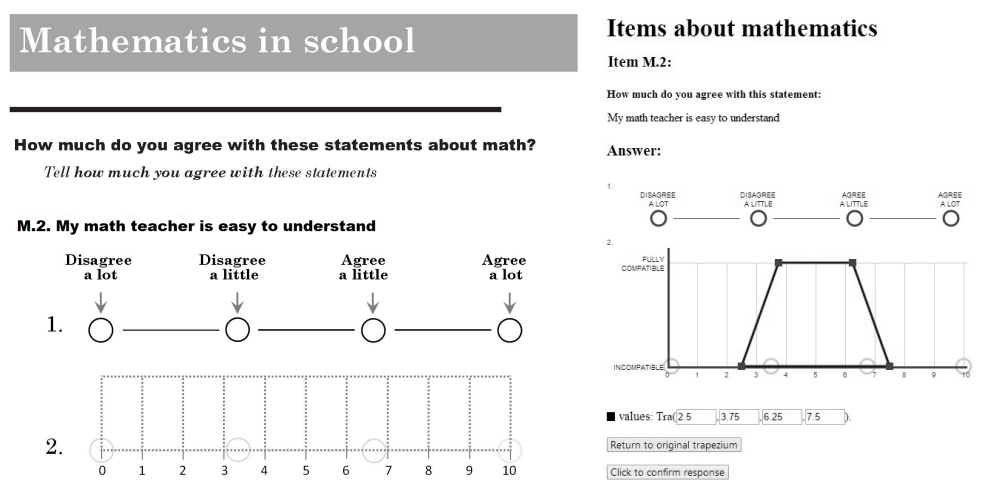

Fig. 2: Example of the double response paper-and-pencil (on the left) and computerized (on the right) form to an item in the case study

The questionnaire involving these double response questions has been conducted in 2014 on a sample of 69 fourth grade students from Colegio San Ignacio (OviedoAsturias, Spain). These students have been distributed in accordance with (their usual) three groups, so that the teachers have decided that the 24 students in one of the three classrooms have to fill out the paper-and-pencil format and the 45 students from the other two groups have to complete the computerized version. To 'ease' the relationship between the two scales for these very young respondents, each numerically encoded Likert response has been superimposed upon the reference interval of the fuzzy rating scale part, as we can see in Figure 2.

The training of the students to let them know about the meaning and purpose of the case study, as well as the aim of the double response, has been carried out in up to 15 minutes, and three researchers from our Department have been in charge of the explanation and conduction of the survey. At this point, it should be remarked that the students had no idea on the concept of real-valued functions and they have just learned that of a trapezium. The students have not had understanding problems, they have catched the philosophy behind and they have been able to provide us with quite coherent responses in most of the cases. Actually, for all the questions, the number of 'no response"s has been very small and smaller for the fuzzy rating than for the Likert scale. In summary, the training has been surprisingly much easier and more effective than we had expected.

The complete questionnaire and dataset can be found in http://bellman. ciencias.uniovi.es/SMIRE/FuzzyRatingScaleQuestionnaire-Sanlgnacio.html. 


\section{Comparative analysis}

The analysis of the influence of fuzzy data shape on the dataset variance is to be based on the test about the equality of variances with fuzzy data developed by Ramos-Guajardo and Lubiano [10] (see also Blanco-Fernández et al. [5]), which is a bootstrapped homoscedasticity test of $k$ independent RFN's, which can be algorithmically summarized.

The analysis is carried out aiming to test the influence of the shape of fuzzy data on the Fréchet variance. By means of some of the data in the considered case study, this section follows two different comparative approaches. More concretely, it is first devoted to compare the $p$-values of two-sample and $k$-sample test about the equality of variances for different choices of the shape.

Table 2 gathers the $p$-values of the two-sample test about the equality of variances on the basis of the fuzzy rating scale responses to Item M.2 in the adapted questionnaire (that is, "My math teacher is easy to understand") when the two considered populations are 'boys' and 'girls' and the 4-tuples are associated not only with trapezoidal fuzzy numbers (as it has actually been made) but also with other $L R$ 's (those in Figure 1 along with $\operatorname{Tri}(a, b, c, d)=\operatorname{Tra}(a,(b+c) / 2,(b+c) / 2, d)$ and $\operatorname{TriS}(a, b, c, d)=\operatorname{Tra}(a,(a+d) / 2,(a+d) / 2, d))$. The $p$-values have been computed for $\theta=1 / 3$ and 1 .

Table 2: $p$-Values for the equality of population Fréchet's variances $(\theta=1 / 3, \theta=1)$ of boys' and girls' $L R$ 's responses to Item $M .2$ in the case study, depending on the considered shape

\begin{tabular}{c||c||c||c||c||c||c||c||c} 
& Tra & $\Pi$ & $L U_{1 A}$ & $L U_{1 B}$ & $L U_{2 A}$ & $L U_{2 B}$ & Tri & TriS \\
\hline \hline $\boldsymbol{\theta}=1 / 3$ & 0.416 & 0.478 & 0.539 & 0.466 & 0.473 & 0.456 & 0.466 & 0.397 \\
\hline \hline $\boldsymbol{\theta}=1$ & 0.414 & 0.443 & 0.512 & 0.452 & 0.467 & 0.456 & 0.450 & 0.376
\end{tabular}

For the usually selected significance levels (those being lower than 0.25 ), there are no significant differences between boys and girls in responding to $M .2$, irrespectively of the considered shape of fuzzy data and even of the choice of $\theta$.

Table 3 gathers the $p$-values of the two-sample test about the equality of variances on the basis of the fuzzy rating scale responses to Item $M .2$ in the case study when the two considered populations are 'paper-and-pencil' and 'computerized' form and the 4-tuples are associated with several $L R$-valued fuzzy numbers. The $p$-values have been computed for $\theta=1 / 3$ and 1 .

Table 3: $p$-Values for the equality of population Fréchet's variances $(\theta=1 / 3, \theta=1)$ of 'paperand-pencil' and 'computerized' form's $L R$ 's responses to Item $M .2$ in the case study, depending on the considered shape

\begin{tabular}{c||c||c||c||c||c||c||c||c} 
& Tra & $\Pi$ & $L U_{1 A}$ & $L U_{1 B}$ & $L U_{2 A}$ & $L U_{2 B}$ & Tri & TriS \\
\hline \hline$\theta=1 / 3$ & 0.215 & 0.220 & 0.239 & 0.217 & 0.233 & 0.198 & 0.234 & 0.190 \\
\hline \hline$\theta=1$ & 0.149 & 0.166 & 0.186 & 0.165 & 0.161 & 0.165 & 0.184 & 0.161
\end{tabular}


In this second situation, the effect of the choice of $\theta \in(0,1]$ is not very relevant. Statistical conclusions scarcely depend on the considered shape of fuzzy data.

Table 4: $p$-Values for the equality of population Fréchet's variances $(\theta=1 / 3, \theta=1)$ of the four groups, $\boldsymbol{G 1}$ to $\boldsymbol{G} 4, L R$ 's responses to Item $M .2$ in the case study, depending on the considered shape

\begin{tabular}{c||c||c||c||c||c||c||c||c} 
& Tra & $\Pi$ & $L U_{1 A}$ & $L U_{1 B}$ & $L U_{2 A}$ & $L U_{2 B}$ & Tri & TriS \\
\hline \hline$\theta=1 / 3$ & 0.270 & 0.255 & 0.255 & 0.282 & 0.263 & 0.275 & 0.247 & 0.218 \\
\hline \hline$\theta=1$ & 0.258 & 0.260 & 0.274 & 0.239 & 0.247 & 0.268 & 0.251 & 0.241
\end{tabular}

Table 4 gathers the $p$-values of the four-sample test about the equality of variances on the basis of the fuzzy rating scale responses to Item M.2 in the case study when the four considered populations are four groups of students based on their 'mark taken in the last examination of math' given by $\boldsymbol{G} \mathbf{1}=[0,6], \boldsymbol{G} \mathbf{2}=(6,8]$, $\boldsymbol{G 3}=(8,9]$ and $\boldsymbol{G} \mathbf{4}=(9,10]$, according to the usual range $[0,10]$ which is considered in Spain. The $p$-values have been computed for $\theta=1 / 3$ and 1 and the 4-tuples are associated with several $L R$-valued fuzzy numbers.

Once more, in this third situation statistical conclusions scarcely depend on the considered shape of fuzzy data.

A second way to analyze the influence of the shape of fuzzy data by means of the case study, is to compare by means of the two-sample test about the equality of variances trapezoidal data $v s$ other $L R$ data in the responses to Item $M .2$ for different populations involved in the preceding tables in this section. Table 5 collects the corresponding $p$-values for $\theta=1 / 3$.

Table 5: $p$-Values for the equality of population Fréchet's variances $(\theta=1 / 3)$ of trapezoidal $v s$ other $L R$ 's responses

\begin{tabular}{c||c||c||c||c||c||c|c} 
Groups $\backslash L R$ & $\Pi$ & $L U_{1 A}$ & $L U_{1 B}$ & $L U_{2 A}$ & $L U_{2 B}$ & Tri & TriS \\
\hline \hline Boys & 0.998 & 0.909 & 0.916 & 0.994 & 0.902 & 0.970 & 0.897 \\
\hline Girls & 1.000 & 0.974 & 0.951 & 0.998 & 0.940 & 0.972 & 0.843 \\
\hline \hline Paper-and-pencil & 0.997 & 0.998 & 0.964 & 0.997 & 0.938 & 0.994 & 0.890 \\
\hline Computerized form & 0.995 & 0.902 & 0.914 & 0.992 & 0.901 & 0.961 & 0.852 \\
\hline \hline $\boldsymbol{G 1}$ & 0.991 & 0.866 & 0.923 & 0.980 & 0.904 & 0.832 & 0.820 \\
\hline $\boldsymbol{G 2}$ & 0.997 & 0.922 & 0.927 & 0.995 & 0.920 & 0.978 & 0.923 \\
\hline $\boldsymbol{G 3}$ & 1.000 & 0.979 & 0.949 & 0.998 & 0.949 & 0.986 & 0.927 \\
\hline $\boldsymbol{G} \mathbf{4}$ & 0.996 & 0.999 & 0.975 & 0.995 & 0.968 & 0.986 & 0.917
\end{tabular}

Consequently, there are no significant differences between population Fréchet's variances for almost all the significance levels one can consider and all the seven developed comparisons. 
Acknowledgements The research in this paper has been partially supported by the Principality of Asturias/FEDER Grant GRUPIN14-101, and the Ministerio de Economía y Competitividad Grants MTM2013-44212-P and MTM2015-63971-P. We acknowledge this financial support.

\section{Authors' dedication}

Dear Pedro,

How difficult is to write a dedication to you!

How difficult assuming there is more than a year since you left us!

How difficult to find the right and accurate words to remember you!; to remember how much you meant (and still mean) for all of us. Words that will be little compared to what you deserve.

How difficult to write a dedication to a very honest man, a good man!

You have been a unique and unrepeatable man, with whom we feel deeply honored to have shared part of our lives.

You have been a generous man, always supporting those who have shared with you the love for Mathematics and, in particular, for Statistics and Operations Research. Fleing from imposition and taxation, you have taught your many disciples the need to devote a significant part of their working time to conduct forefront research. This vision and policy, which can seem to be obvious nowadays, was especially laudable at the time you create the embryo of our current university department.

Beyond your professional stature, your stature as a human being has been even greater. You have been permanently available to all your colleagues and students, willing to help and advice with a warm attitude. In each scenario, you have been always there to provide them with your calm, thoughtful and insightful opinion.

Pedro, you have been a good man, even in the discrepancy. In a rather hierarchical world, where disagreement can often entail a certain degree of punishment or discrimination of the weakest members, you have never made use of your heading position in such an unfair way.

So, in remembering you, we necessarily have to say that you have been the good friend, the magnificent researcher and teacher, and the wonderful colleague we all would like to be. The type of person all of us are delighted and honored to have found along our lives. We only regret that our joint life has not lasted longer.

Oviedo, August 2017 
Fuzzy data shape and Fréchet-type variance

\section{References}

1. Bertoluzza C, Corral N, Salas A (1995) On a new class of distances between fuzzy numbers. Math \& Soft Comput 2:71-84

2. Blanco-Fernández A, Casals MR, Colubi A, Corral N, García-Bárzana M, Gil MA, GonzálezRodríguez G, López MT, Lubiano MA, Montenegro M, Ramos-Guajardo AB, De la Rosa de Sáa S, Sinova B (2014) A distance-based statistical analysis of fuzzy number-valued data. Int J Approx Reas 55(7):1487-1501

3. Dubois D, Prade H (1981) Additions of interactive fuzzy numbers. IEEE Trans Automat Contr 26:926-936

4. Gil MA, Lubiano MA, De la Rosa de Sáa S, Sinova B (2015) Analyzing data from a fuzzy rating scale-based questionnaire. A case study. Psicothema 27:182-191

5. Hesketh T, Pryor R, Hesketh B (1988) An application of a computerized fuzzy graphic rating scale to the psychological measurement of individual differences. Int J Man-Mach Stud. 29:21-35

6. Lubiano MA, Gil MA, López-Díaz M, López MT (2000) The $\boldsymbol{\lambda}$-mean squared dispersion associated with a fuzzy random variable. Fuzzy Sets Syst 111:307-317

7. Lubiano MA, Montenegro M, Sinova B, De la Rosa de Sáa S, Gil MA (2016) Hypothesis testing for means in connection with fuzzy rating scale-based data: algorithms and applications. Eur J Oper Res 251:918-929

8. Lubiano MA, Salas A, Gil MA (2017) A hypothesis testing-based discussion on the sensitivity of means of fuzzy data with respect to data shape. Fuzzy Sets Syst (in press, doi:10.1016/j.fss.2016.10.015)

9. Puri ML, Ralescu DA (1986) Fuzzy random variables. J Math Anal Appl 114:409-422

10. Ramos-Guajardo AB, Lubiano MA (2012) $K$-sample tests for equality of variances of random fuzzy sets. Comp Stat Data Anal 56(4):956-966

11. Stefanini L, Sorini L, Guerra ML (2006) Parametric representation of fuzzy numbers and applications to fuzzy calculus. Fuzzy Sets Syst 157:2423-2455

12. Trutschnig W, Lubiano MA. SAFD: Statistical Analysis of Fuzzy Data (http://cran.rproject.org/web/packages/SAFD/index.html)

13. Zadeh LA (1975) The concept of a linguistic variable and its application to approximate reasoning. Part 1. Inform Sci 8:199-249; Part 2. Inform Sci 8:301-353; Part 3. Inform Sci 9:43-80 